\title{
3D Nested Distributed Massive MIMO: Modeling and Performance Analysis
}

\author{
Shitong Yuan, Qilian Liang \\ Department of Electrical Engineering \\ University of Texas at Arlington \\ Arlington, TX-76019-0016 \\ Email:shitong.yuan@mavs.uta.edu, liang@uta.edu
}

\begin{abstract}
The Large Scale Multiple-input-multiple-output (MIMO) system, also called Massive MIMO in past researches, are considering the azimuth angle only. However, in a 3D distributed antenna system, the elevation angle cannot be ignored. Nested array as a two dimensional arrays was firstly proposed to perform array processing with increased degree of freedom, using less number of sensors at the same time. This paper introduces a novel 3D MIMO antenna deployment based on nested co-array. As the difference co-arrays are invariance, in the 3D nested distributed MIMO system, we are able to calculate the covariance matrix of channels. Based on this inference, we model a 3D nested distributed MIMO system and analyze its performance with achievable sum rate. By applying the nested deployment, our proposed method could achieve a Massive MIMO with less number of physical antennas. Numerical results also provided to support our model.
\end{abstract}

Index Terms-3D, Massive MIMO, Difference Co-array, Nested Array.

\section{INTRODUCTION}

4G LTE technology has been released to the market several years ago. However, some new challenges emerged: on one hand mobile devices and Internet services are developing quickly-people watching better quality video stream and sending much more photo messages; On the other hand, more people start using $4 \mathrm{G}$ services especially in developing countries with huge population. Then there is a shortage of wireless resources. Generally, there are two possible ways to solve this problem: Allocate more frequency band to cellular systems; Improve the spectral efficiency to get better capacity. So in next generation communication system, which is expected to be in use around 2020 [20], people plan to use the frequency up to $50 \mathrm{GHz}$ [22]. Some new technologies are also expected in the next generation cellular system, such as device-to-device (D2D) [21], ultra-dense networks (UDNs) [1] and Massive MIMO [12] [10].

Massive MIMO is one of the most popular areas and many researches have been done on it. In [5], a method to evaluate the performance of Massive MIMO system was proposed. Trellis-extended codebooks for Massive MIMO was introduced by [2]. The achievable rates of spatial channel correlation FDD Massive MIMO systems was investigated in [9].
3D MIMO system is an important topic in 5G. Because consider the physical characteristics of $50 \mathrm{GHz}$ electromagnetic waves, Distributed antenna system (DAS) [3] and massive MIMO technologies could be utilized to overcome the affect of the high wallpenetration losses [23]. A 3D Massive MIMO system modeling and performance analysis was been done in [15]. A novel theoretical non-stationary three dimensional (3-D) wideband twin-cluster channel model for massive MIMO was proposed in [24]. However, there are still several intrinsic problems for Massive MIMO system, particularly in three-dimensional. The densely large number of antennas may result in serious interference.

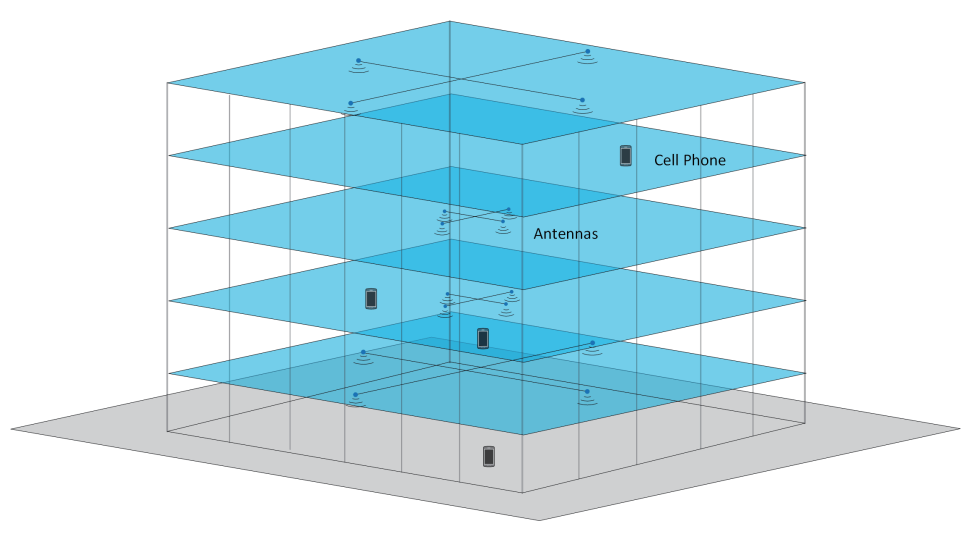

Fig. 1. 3D Nested Distributed MIMO System in A Building

This paper introduces a novel 3D Massive MIMO antenna network. This system is proposed to facilitate the study of Massive MIMO antenna 3D deployment and the cooperation between antennas. This is the first manuscript on modeling a 3D MIMO cellular system with nested distributed antennas. Nested array was initially proposed as a novel approach to enhance the degrees of freedom of an array[17]. Following that, [18][19] exhaustive discussed and studied the sensors' placement and degree of freedom analysis with nested-array configuration, which use much less sensors to achieve the similar performance.

The rest of this paper is organized as follows. Section II briefly introduces the theoretical background of two dimen- 
sional nested array. In Section III, an antenna pair with 3D cross difference co-array configuration is modeled. Section IV introduce the modeling of 3D nested deployed MIMO antenna network. It also illustrates how to calculate sum rate capacity. After that, in Section V some numerical results are provided to demonstrate accuracy of our proposed scheme. Finally, we summarize our work and draw conclusions in the last Section.

\section{Preliminary Work AND 3D Nested Array}

Firstly, let us review several important concepts related to multi-dimensional lattice and nested array, which are necessary for deriving our Massive MIMO placement method.

Definition 1: (Fundamental Parallelepiped(FPD) [18]) The FPD is defined as the set of all vectors of the following form:

$$
F P D(\mathbf{V})=\left\{\mathbf{V} \mathbf{x}, \mathbf{x} \in\left[\begin{array}{ll}
0 & 1
\end{array}\right)^{D}\right\}
$$

Where $\mathbf{V} \in C^{D \times D}$. Apparently, the $\operatorname{FPD}(\mathbf{V})$ contains all points inside the parallelepiped and its two sides are actually given by two columns vectors of $\mathbf{V}$. The volume of $\operatorname{FPD}(\mathbf{V})$ is given by $\|\operatorname{det}(\mathbf{V})\|$, it represents the area in $2-\mathrm{D}$ cases. And $1 /\|\operatorname{det}(\mathbf{V})\|$ is the density of arrays(antennas) correspondingly.

Definition 2: (Shift FPD(SFPD)) Consider $k_{1}, k_{2}$ as two integers, the $F P D\left(N^{(s)}\right)$ shifted by $\left[k_{1}, k_{2}\right]^{T}$ could obtain $S F P D\left(N^{(s)}\right)$, mathematically could be defined as:

$$
\operatorname{SFPD}\left(N^{(s)}, k_{1}, k_{2}\right) \equiv\left\{N^{(s)}\left(\left[k_{1}, k_{2}\right]^{T}-x\right), x \in[0,1)^{2}\right\} .
$$

Definition 3: (Cross Difference Co-array) let us consider an array of $N$ antennas, with $\vec{v}_{n}$ denoting the position of n-th sensor(antenna). Then the difference co-array could be written as:

$$
\left\{\vec{v}_{n}-\vec{v}_{m}\right\}, \forall m, n=1,2, \ldots, N .
$$

Based on difference co-array, the cross difference co-array could be considered as: given two arrays, one with $N_{1}$ sensors with locations $\left\{\vec{v}_{1 i}, 1 \leq i \leq N_{1}\right\}$ and the other with $N_{2}$ sensors with locations $\left\{\vec{v}_{2 i}, 1 \leq i \leq N_{2}\right\}$, their cross difference co-array is defined as the set:

$$
\pm\left\{\vec{v}_{1 i}-\vec{v}_{2 i}, 1 \leq i \leq N_{1}, 1 \leq i \leq N_{2}\right\}
$$

Definition 4: (Two Dimensional Nested Array) A $2 \times 2$ non-singular matrix $\mathbf{N}^{(d)}$, an integer matrix $\boldsymbol{P}$ and integers $N^{(s)}$ can be used to describe a two dimensional nested array.

1) A dense array generated by $\mathbf{N}^{(d)}$ with $N^{(d)}=\operatorname{det}[P]$ elements on this dense lattice. And the sensors' locations given by $\left\{\mathbf{N}^{(d)} \mathbf{n}^{(d)}, \mathbf{n}^{(d)} \in F P D(P)\right\}$.

2) A sparse array generated by $\mathbf{N}^{(s)}=\mathbf{N}^{(d)} \mathbf{P}$ with antennas' location given by:

$\left\{\mathbf{N}^{(s)}\left[k_{1}, k_{2}\right]^{T}, 0 \leq k_{1} \leq N_{1}^{(s)}-1,0 \leq k_{2} \leq N_{2}^{(s)}-1\right\}$.

Theorem 1: Consider two non-singular $2 \times 2$ matrices $\mathbf{N}^{(s)}$ and $\mathbf{N}^{(d)}$ related by integer $\mathbf{P}$ as $\mathbf{N}^{(s)}=\mathbf{N}^{(d)} \mathbf{P}$.
1) Any point on dense lattice $\mathbf{N}^{(d)}$ has a location vector denoted as $\mathbf{N}^{(d)} \mathbf{n} . \mathbf{N}^{(d)} \mathbf{n}=\mathbf{N}^{(s)} \mathbf{n}^{(s)}-\mathbf{N}^{(d)} \mathbf{n}^{(d)}$, where $\mathbf{n}^{(s)}$ is an integer vector and $\mathbf{n}^{(d)} \in F P D(\mathbf{P})$.

2) All points within $\operatorname{SFPD}\left(\mathbf{N}^{(s)}, k_{1}, k_{2}\right)$ can be generated by the differences:

$\left\{\mathbf{N}^{(s)}\left[k_{1}, k_{2}\right]^{T}-\mathbf{N}^{(d)} \mathbf{n}^{(d)}, \mathbf{n}^{(d)} \in F P D(\mathbf{P})\right\}$.

Based on the 2D Nested Co-array, we can easily derive the 3D Nested Co-array. First, the SFPD could be considered as a FPD shifted by a vector $\left[k_{1}, k_{2}, k_{3}\right]$. And then, $\mathbf{N}^{(d)}$ becomes a $3 \times 3$ non-singular matrix. An example of $3 \mathrm{D}$ Nested MIMO system will be illustrate in the end of next section.

Definition 5: (Three Dimensional Nested Array) A $3 \times 3$ non-singular matrix $\mathbf{N}^{(d)}$, an integer matrix $\mathbf{P}$ (Expanded to $3 \times 3$ as well) and integers $N^{(s)}$ can be used to describe a three dimensional nested array following Definition 4.

\section{System AND Channel Model}

According to the definition of cross difference co-array and Shift FPD, virtual antennas' location could be decided by any two physical antennas. So the problem we study here becomes: Given physical antennas (some of them are densely deployed, some are sparsely deployed), and their location known and fixed, channels established and channel coefficients known, how to locate virtual antennas and obtain virtual channels based on Nested array.

1) Difference Co-array of Two Antennas: Consider a nested antenna array with maximum $M=2 N_{1}^{(s)} N_{2}^{(s)} N_{3}^{(s)}-2$ antennas on the sparse lattice generated by $\mathbf{N}^{(s)}$. And $N=\operatorname{det}(\mathbf{P})$ antennas on dense lattice obtained by generation matrix $\mathbf{N}^{(d)}$. Following definitions in last section, we can denoting the locations of antennas with $\mathbf{N}^{(s)} \mathbf{n}^{(s)}$ for sparse antennas array and $\mathbf{N}^{(d)} \mathbf{n}^{(d)}$ for dense antennas. Where $\mathbf{n}^{(s)}$ and $\mathbf{n}^{(d)}$ are integer vectors, $\mathbf{n}^{(d)} \in \operatorname{FPD}(\mathbf{P})$.

We assume that there are $K$ users in this system. Based on Definition 3, any two physical antennas can become a difference co-array pair. We take one antenna from sparse array and the other one from dense array in the following modeling. Then the signal received at one of sparse array antennas $m$ form user $k$ is:

$$
y_{m}^{U L}[k]=h_{k, m}^{U L} e^{j \omega_{k}^{m} \sin \phi_{k}^{m} v_{m}} x^{U L}[k]+\eta_{0}
$$

Where, $\omega_{k}^{m}=\frac{2 \pi}{\lambda}\left[\cos \theta_{k}^{m} \sin \theta_{k}^{m}\right], \lambda$ is the signal wave length. $\theta_{k}^{m}$ is the azimuthal angle from k-th users to $\mathrm{m}$-th antenna. $\phi_{k}^{m}$ is the elevation angle correspondingly.

$\overrightarrow{v_{m}}=\mathbf{N}^{(s)} \mathbf{n}_{m}^{(s)}$ is the location of $\mathrm{m}$-th antenna in the 3D Cartesian coordinate system. $\mathbf{n}_{m}^{(s)}$ is an $3 \times 1$ integer vector.

Similarly, the signal received at one of dense array antennas $n$ from the same user $k$ can be written as:

$$
y_{n}^{U L}[k]=h_{k, n}^{U L} e^{j \omega_{k}^{n} \sin \phi_{k}^{n} \vec{v}_{n}} x^{U L}[k]+\eta_{0}
$$




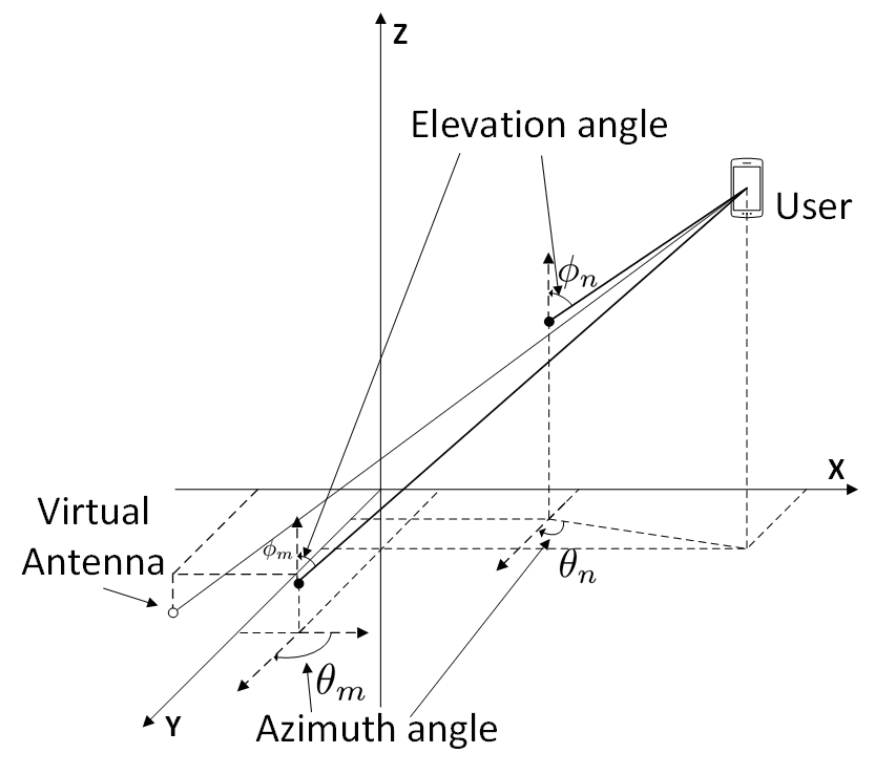

Fig. 2. A 3D Virtual Antenna Generated by A Pair of Difference Co-Array

The same, $\omega_{k}^{n}=\frac{2 \pi}{\lambda}\left[\cos \theta_{k}^{n} \sin \theta_{k}^{n}\right]$, and $\overrightarrow{v_{n}}=\mathbf{N}^{(d)} \mathbf{n}_{n}^{(d)}$. Then it is ready to derive the cross correlation between the signal received at $\mathrm{m}$-th and $\mathrm{n}$-th antenna from the same source user k:

$$
\begin{aligned}
& E\left[y_{n}^{U L}[k]\left(y_{m}^{U L}[k]\right)^{H}\right]= \\
& \quad h_{k, n}^{U L} e^{j\left(\omega_{k}^{n} v_{n} \sin \phi_{k}^{n}-\omega_{k}^{m} v_{m} \sin \phi_{k}^{m}\right)} x^{U L}[k]\left(x^{U L}[k]\right)^{H}\left(h_{k, m}^{U L}\right)^{H} \\
& \quad+\sigma^{4} \mathbf{e}_{m, n} \mathbf{e}_{m, n}^{H}+\sigma^{2} h_{k, n}^{U L} e^{j \omega_{k}^{n} \sin \phi_{k}^{n} \overrightarrow{v_{n}}} x^{U L}[k] \mathbf{e}_{m, n}^{H} \\
& \quad+\sigma^{2} \mathbf{e}_{m, n}\left(h_{k, m}^{U L}\right)^{H} e^{j \omega_{k}^{m} \sin \phi_{k}^{m} \overrightarrow{v_{m}}}\left(x^{U L}[k]\right)^{H}
\end{aligned}
$$

The elements $\omega_{k}^{n} \sin \phi_{k}^{n} \overrightarrow{v_{n}}-\omega_{k}^{m} \sin \phi_{k}^{m} \overrightarrow{v_{m}}$ here in the exponents of cross difference co-array terms behave like the signal received by a virtual antenna. The virtual antenna's location is decided by signals' arrival azimuthal angle, elevation angle and locations of physical antennas. Based on the mathematical relation above, we have:

Proposition 1. The possible maximum number of virtual antennas is $N(N-1)+1$, where $N$ is the total number of physical antennas.

Proposition 2. If $\vec{v}=\omega_{k}^{n} \sin \phi_{k}^{n} \overrightarrow{v_{n}}-\omega_{k}^{m} \sin \phi_{k}^{m} \overrightarrow{v_{m}}$ denotes the location of one virtual antenna, then there must be a virtual antenna locates at $-\vec{v}$ (They are Central symmetric about $\mathbf{0}$ ).

So it is possible to generate as many as $N(N-1)+1$ virtual antennas with optimized and appropriate physical antenna placement. Since the difference co-array is symmetric about $\mathbf{0}$, we can design the physical antenna distribution to get desired coverage and performance.
The downlink of this system has similar form compare with the uplink. Based on equation (2)(3), we can write the signal transmit from m-th sparse array antenna to k-th user as:

$$
y_{m}^{D L}[k]=h_{k, m}^{D L} e^{j \omega_{k}^{m} \sin \phi_{k}^{m} v_{m}} x^{D L}[k]+\eta_{m}[k]
$$

And the signal transmit from $\mathrm{n}$-th dense array antenna to $\mathrm{k}$-th user is:

$$
y_{n}^{D L}[k]=h_{k, n}^{D L} e^{j \omega_{k}^{n} \sin \phi_{k}^{n} \vec{v}_{n}} x^{D L}[k]+\eta_{n}[k]
$$

For the downlink, signals have experienced the different distortion from different antennas [6]. So we can derive the cross correlation between those two signals from $\mathrm{m}$-th and n-th antenna as:

$$
\begin{aligned}
& E\left[y_{n}^{D L}[k]\left(y_{m}^{D L}[k]\right)^{H}\right]= \\
& \quad h_{k, m}^{D L} e^{j\left(\omega_{k}^{n} \overrightarrow{v_{n}} \sin \phi_{k}^{n}-\omega_{k}^{m} \vec{v}_{m} \sin \phi_{k}^{m}\right)} x^{D L}[k]\left(x^{D L}[k]\right)^{H}\left(h_{k, m}^{D L}\right)^{H} \\
& \quad+\sigma_{n}^{2} \sigma_{m}^{2} \mathbf{e}_{m, n}^{H}+\sigma_{n}^{2} h_{k, n}^{D L} e^{j \omega_{k}^{n} \sin \phi_{k}^{n} \overrightarrow{v_{n}}} x^{D L}[k] \mathbf{e}_{m, n}^{H} \\
& \quad+\sigma_{m}^{2} \mathbf{e}_{m, n}\left(h_{k, m}^{D L}\right)^{H} e^{j \omega_{k}^{m} \sin \phi_{k}^{m} v_{m}}\left(x^{D L}[k]\right)^{H}
\end{aligned}
$$

\section{A. An Example of 3D Nested Massive MIMO System}

An example in Fig. 3 shows a 3D nested deployed Massive MIMO system with $\mathbf{N}^{(d)}=\left(\begin{array}{lll}1 & 0 & 0 \\ 0 & 1 & 0 \\ 0 & 0 & 1\end{array}\right)$ and $\mathbf{P}=$ $\left(\begin{array}{lll}2 & 0 & 0 \\ 0 & 2 & 0 \\ 0 & 0 & 2\end{array}\right)$. According to the definitions of nested array, there are $|\mathbf{P}|=N^{(d)}=8$ antennas densely placed. In this case, we assume $N_{1}^{(s)}=3, N_{2}^{(s)}=3, N_{3}^{(s)}=3$, so there are $2 N_{1}^{(s)} N_{2}^{(s)} N_{3}^{(s)}-2=52$ antennas sparsely deployed.By applying the difference co-array, 281 virtual antennas are generated by $8+52=60$ physical antennas.

\section{Achievable Rate of 3D Nested Massive MiMO SYSTEM}

\section{A. Channel Parameters}

In a huge building, both users and distributed antennas are created across different heights (due to different floors), not just at ground level as 2D cases. If we do not consider the height of building, then the user's height $h_{U E}=1.6 \mathrm{~m}$. In 3D scenario, users are randomly distributed with height $h_{U E}=$ $\left(n_{f l}-1\right) \times 3+1.6 m$, where $n_{f l}$ is the number of floors and we assume height of each floor is $3 \mathrm{~m}$. The existing Path Loss for Line of Sight(LOS) and Non-LOS models need some modifications to fit the 3D Massive MIMO scenarios. Based on [7][8], the modified Pass Loss are as follows:

If $3 m \leq d_{3 D} \leq d_{B P}$

$$
P L_{L O S}=16.9 \log _{10}\left(d_{3 D}\right)+32.8+20 \log _{10}\left(f_{c}\right),
$$

Otherwise,

$$
\begin{aligned}
P L_{L O S}= & 40 \log _{10}\left(d_{3 D}\right)+28+ \\
& 20 \log _{10}\left(f_{c}\right)-9 \log _{10}\left(d_{B P}^{2}+\left(h_{B S}-h_{U E}\right)^{2}\right)
\end{aligned}
$$




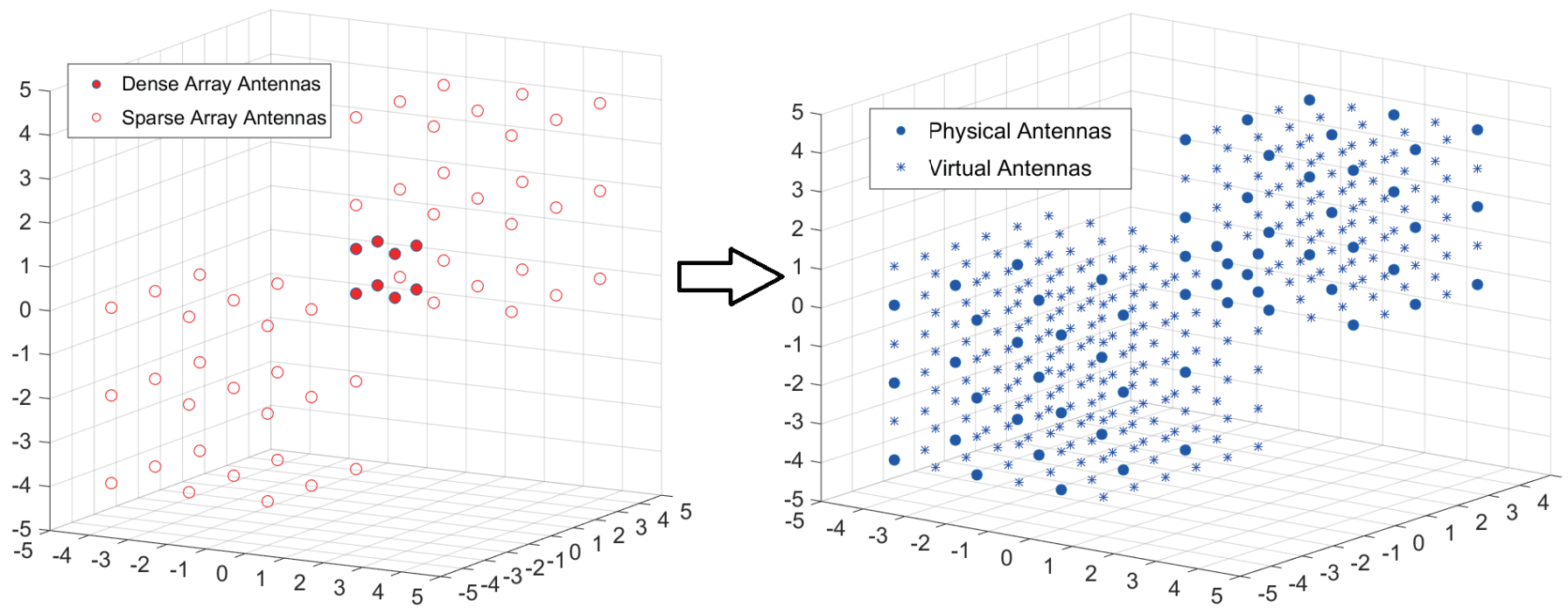

Fig. 3. An Example of 3D nested Antenna Array

where $f_{c}$ is the central frequency given in $\mathrm{GHz}$. $d_{B P}=2 \pi h_{B S} h_{U E} \frac{f_{c}}{c}$ is the break point distance. For the non Line of Sight, similarly we have:

$$
\begin{aligned}
P L_{N L O S}= & 43.3 \log _{10}\left(d_{3 D}\right)+11.5+ \\
& 20 \log _{10}\left(f_{c}\right)-0.6\left(h_{U E}-1.6\right)
\end{aligned}
$$

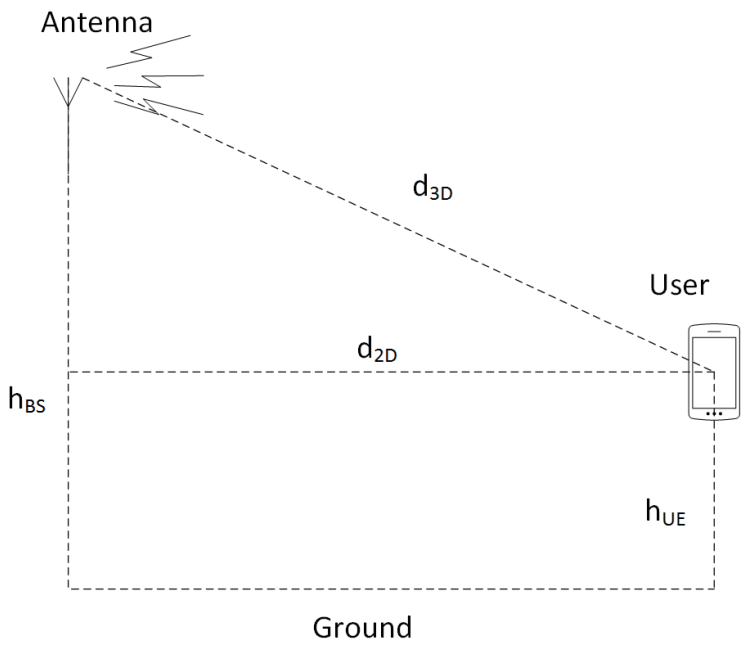

Fig. 4. 3D Large Scale Parameters

In addition to just consider the height of antennas and users, the elevation angles of antenna bore sight also affect the performance. This angle, we say $\phi_{t i l t}$, usually is assumed to be fixed at $\frac{\pi}{2}$ in $2 \mathrm{D}$ scenarios. However, as measured and proved in some papers [15][11][4], there are some energy propagate in the elevation which can not be ignored. Considering the azimuth angle alone here in $3 \mathrm{D}$ case is not accurate any more. Also if we fix the antenna bore sight, channels' degree of freedom in the elevation is wasted. In [7],
ITU (International Telecommunication Union) approximates the horizontal antenna pattern as:

$$
A_{h}(\theta)=-\min \left[12\left(\frac{\theta}{\theta_{3 d B}}\right)^{2}, A_{m}\right]
$$

Where $A_{h}(\theta)$ is the antenna gain $(\mathrm{dB})$ in this direction, $\theta \in$ $[-\pi, \pi], \theta_{3 d B}$ is the $3 \mathrm{~dB}$ beam-width (Usually $\theta_{3 d B}=70^{\circ}$ in practice). And $A_{m}=20 d B$ is the maximum attenuation. The vertical antenna pattern as:

$$
A_{e}(\phi)=-\min \left[12\left(\frac{\phi-\phi_{t i l t}}{\phi_{3 d B}}\right)^{2}, A_{m}\right]
$$

Where $A_{e}(\phi)$ is the antenna gain $(\mathrm{dB})$ in elevation direction, $\theta \in\left[-\frac{\pi}{2}, \frac{\pi}{2}\right], \phi_{\text {tilt }}$ is the tilt angle, it may be assumed that $\phi_{3 d B}=15^{\circ}$.

The combined antenna pattern at angles off the cardinal axes can be written as:

$$
A_{h, e}(\theta, \phi)=-\min \left[-\left(A_{h}(\theta)+A_{e}(\phi)\right), A_{m}\right]
$$

\section{B. Model of 3D Nested MIMO System}

Based on the derivation in previous sections, we can write the output of receiver with matrix form as:

$$
\mathbf{Y}[k]=\left(\begin{array}{l}
\mathbf{H}_{s} \\
\mathbf{H}_{d}
\end{array}\right) \mathbf{X}[k]+\eta[k]
$$

Where $\mathbf{H}_{d}$ is a $\operatorname{det}(\mathbf{P}) \times K$ matrix for dense array and $\mathbf{H}_{s}$ is a $\left(2 N_{1}^{(s)} N_{2}^{(s)} N_{3}^{(s)}-2\right) \times K$ matrix for sparse array. $\mathbf{X}[k]$ is the source signal vector and the AWGN with power $\sigma^{2}$ is represented as $\eta[k]$.

For $\mathbf{H}_{d}$, its elements are:

$$
\left.\left[\mathbf{H}_{d}\right]_{t, k}=h_{t, k} e^{j \frac{2 \pi}{\lambda} \sin \phi_{k}^{t}\left[\cos \theta_{k}\right.} \sin \theta_{k}\right]^{T} \mathbf{N}^{(d)} \mathbf{n}_{t}^{(d)}
$$


Where $k=1,2, \ldots, K$ and $t=1,2, \ldots, \operatorname{det}(\mathbf{P})$. Similarly, the elements of $\mathbf{H}_{s}$ are:

$$
\left[\mathbf{H}_{s}\right]_{t, k}=h_{t, k} e^{j \frac{2 \pi}{\lambda} \sin \phi_{k}^{t}\left[\cos \theta_{k} \sin \theta_{k}\right]^{T} \mathbf{N}^{(s)} \mathbf{n}_{t}^{(s)}}
$$

Where $k=1,2, \ldots, K$ and $t=1,2, \ldots, 2 N_{1}^{(s)} N_{2}^{(s)} N_{3}^{(s)}-2$. Then it is ready to derive to auto correlation of the received signal matrix as:

$$
\mathbf{R}_{Y Y}=E\left[\mathbf{Y} \mathbf{Y}^{H}\right]=\left(\begin{array}{c}
\mathbf{H}_{s} \\
\mathbf{H}_{d}
\end{array}\right) \mathbf{R}_{X X}\left(\begin{array}{c}
\mathbf{H}_{s} \\
\mathbf{H}_{d}
\end{array}\right)^{H}+\sigma_{n}^{2} \mathbf{I}
$$

Next step is to vectorize $\mathbf{R}_{Y Y}$. According to the matrix theory, vectorization is a linear operation that means:

$$
V e c\left(k_{1} \mathbf{A}+k_{2} \mathbf{B}\right)=k_{1} V e c(\mathbf{A})+k_{2} V e c(\mathbf{B})
$$

Where $k_{1}$ and $k_{2}$ are parameters. And also vectorization has a following important property:

$$
V e c(\mathbf{A B C})=\left(\mathbf{C}^{T} \otimes \mathbf{A}\right) V e c(\mathbf{B})
$$

Where $\otimes$ denotes the Kronecker product(also called direct product or tensor product), which is defined as:

$$
\mathbf{A} \otimes \mathbf{B}=\left[\begin{array}{ccc}
A_{11} \mathbf{B} & \cdots & A_{1 n} \mathbf{B} \\
\cdot & \cdot & \cdot \\
\cdot & \cdot & \cdot \\
\cdot & \cdot & \cdot \\
A_{m 1} \mathbf{B} & \cdots & A_{m n} \mathbf{B}
\end{array}\right]
$$

Now we are ready to write the vectorized $\mathbf{R}_{Y Y}$ as:

$$
\operatorname{Vec}\left(\mathbf{R}_{Y Y}\right)=\left(\begin{array}{l}
\mathbf{H}_{s} \\
\mathbf{H}_{d}
\end{array}\right)^{*} \otimes\left(\begin{array}{c}
\mathbf{H}_{s} \\
\mathbf{H}_{d}
\end{array}\right) \widetilde{\mathbf{x}}+\sigma_{n}^{2} \widetilde{\mathbf{I}}
$$

Here, $\widetilde{\mathbf{x}}$ is a $K \times 1$ column vector as $\widetilde{\mathbf{x}}=\left[\sigma_{1}^{2}, \ldots, \sigma_{K}^{2}\right]$. $\widetilde{\mathbf{I}}$ is still a diagonal matrix with all 1 , but it size becomes larger to $\left(2 N_{1}^{(s)} N_{2}^{(s)} N_{3}^{(s)}+\operatorname{det}(\mathbf{P})-2\right)^{2}$. Let

$$
\mathbf{H}_{3 D}=\left(\begin{array}{l}
\mathbf{H}_{s} \\
\mathbf{H}_{d}
\end{array}\right)^{*} \otimes\left(\begin{array}{l}
\mathbf{H}_{s} \\
\mathbf{H}_{d}
\end{array}\right)
$$

$\mathbf{H}_{3 D}$ has $K$ columns. It contains all rows $h_{t, l}$ and $h_{t, l}^{*}$ where

$$
\begin{aligned}
& \vec{h}_{t, l}=\left[h_{t, l} e^{j \frac{2 \pi}{\lambda}\left[\cos \theta_{1}\right.} \sin \theta_{1}\right]^{T}\left(\sin \phi_{1}^{t} \mathbf{N}^{(s)} \mathbf{n}_{t}^{(s)}-\sin \phi_{1}^{l} \mathbf{N}^{(d)} \mathbf{n}_{l}^{(d)}\right) \ldots
\end{aligned}
$$

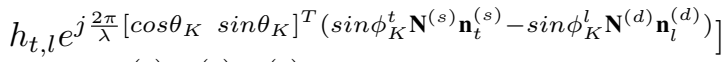

$$
\begin{aligned}
& t=1, \ldots,\left(2 N_{1}^{(s)} N_{2}^{(s)} N_{3}^{(s)}-2\right), l=1, \ldots, \operatorname{det}(\mathbf{P})
\end{aligned}
$$

Those rows are actually generated by a pair of antennas which one from dense array, the other one from sparse array. And also rows $h_{t, l}^{(s)}, h_{t, l}^{*(s)}, h_{t, l}^{(d)}$ and $h_{t, l}^{*(d)}$ where

$$
\begin{aligned}
& \vec{h}_{t, l}^{(s)}=\left[h_{t, l} e^{j \frac{2 \pi}{\lambda}\left[\cos \theta_{1}\right.} \sin \theta_{1}\right]^{T}\left(\sin \phi_{1}^{t} \mathbf{N}^{(s)} \mathbf{n}_{t}^{(s)}-\sin \phi_{1}^{l} \mathbf{N}^{(s)} \mathbf{n}_{l}^{(s)}\right) \ldots
\end{aligned}
$$

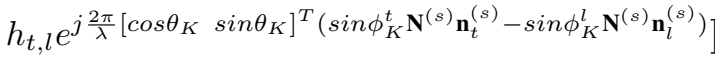

$$
\begin{aligned}
& t, l=1, \ldots, 2 N_{1}^{(s)} N_{2}^{(s)} N_{3}^{(s)}-2
\end{aligned}
$$

The rows above are generated by two antennas from sparse array.

$$
\begin{aligned}
& \vec{h}_{t, l}^{(s)}=\left[h_{t, l} e^{j \frac{2 \pi}{\lambda}\left[\cos \theta_{1}\right.} \sin \theta_{1}\right]^{T}\left(\sin \phi_{1}^{t} \mathbf{N}^{(d)} \mathbf{n}_{t}^{(d)}-\sin \phi_{1}^{l} \mathbf{N}^{(d)} \mathbf{n}_{l}^{(d)}\right) \ldots
\end{aligned}
$$

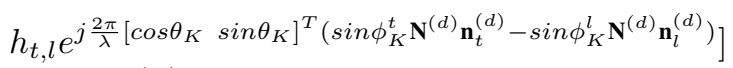

$$
\begin{aligned}
& t, l=1, \ldots, \operatorname{det}(\mathbf{P})
\end{aligned}
$$

The rows above are generated by two antennas from dense array. These rows and their conjugates together in the matrix $\mathbf{H}_{3 D}$ behave exactly like the Massive MIMO channel based on a large 3-D nested antenna array generated by their difference co-array. Then we can write the system model as:

$$
\mathbf{Y}=\mathbf{H}_{3 D} \widetilde{\mathbf{x}}+\sigma_{n}^{2} \widetilde{\mathbf{I}}
$$

\section{Achievable Sum Rate Analysis}

As mentioned before, we suppose there are $K$ users in this system. The channel matrix $\mathbf{H}_{3 D}=\mathbf{G}$ has $M$ rows (means the system has $\mathrm{M}$ physical plus virtual antennas). And we can also assume that both $M, K$ are large numbers and $1 \ll K \ll M$.

Then we could derive the achievable sum rate for this nested distributed Massive MIMO system as:

$$
C_{\text {sum } U L}=\log _{2}\left|\mathbf{I}_{K}+\frac{p_{u}}{K} \mathbf{G}_{u}^{H} \mathbf{G}_{u}\right|
$$

Considering that the system has perfect CSI, i.e. $\mathbf{G}$ is known. $\mathbf{g}_{k}$ is k-th columns which denotes the channel between $\mathrm{k}$-th user and all antennas (both physical and virtual). Then the ergodic achievable Uplink rate of k-th user can be written as:

$$
\begin{aligned}
R_{k} & =E\left\{\log _{2}\left(1+\frac{p_{u}\left\|\mathbf{g}_{k}^{H} \mathbf{g}_{k}\right\|^{2}}{p_{u} \sum_{i=1, i \neq k}^{K}\left\|\mathbf{g}_{k}^{H} \mathbf{g}_{i}\right\|^{2}+\left\|\mathbf{g}_{k}\right\|^{2}}\right)\right\} \\
& =E\left\{\log _{2}\left(1+\frac{p_{u}\left\|\mathbf{g}_{k}\right\|^{4}}{p_{u} \sum_{i=1, i \neq k}^{K}\left\|\mathbf{g}_{k}^{H} \mathbf{g}_{i}\right\|^{2}+\left\|\mathbf{g}_{k}\right\|^{2}}\right)\right\}
\end{aligned}
$$

Based on equations above, we can derive the lower bound of achievable rate of 3D nested deployed Massive MIMO system as [16]:

$$
\begin{aligned}
R_{k} & \geq R_{k}^{L} \\
& =\log _{2}\left(1+\left(E\left\{\frac{p_{u} \sum_{i=1, i \neq k}^{K}\left\|\mathbf{g}_{k}^{H} \mathbf{g}_{i}\right\|^{2}+\left\|\mathbf{g}_{k}\right\|^{2}}{p_{u}\left\|\mathbf{g}_{k}\right\|^{4}}\right\}\right)^{-1}\right) \\
& \geq \tilde{R}_{k}^{L}=\log _{2}\left(1+\frac{p_{u}(M-1) \beta_{k}\left(\phi_{\text {tilt }}\right)}{p_{u} \sum_{i=1, i \neq k}^{K} \beta_{i}\left(\phi_{\text {tilt }}\right)+1}\right) \\
& \stackrel{M}{\rightarrow \infty} \log _{2}\left(1+\beta_{k}\left(\phi_{\text {tilt }}\right) E_{u}\right)
\end{aligned}
$$

Where $p_{u}$ is the average transmitted power of each user, $E_{u}=M \times p_{u}$. If we assume $G_{\max }$ is the maximum antenna gain (in $\mathrm{dB}$ ) at the antenna boresight, based on (11), (12) and [13], the antenna gain in linear scale for k-th user can be 
written as:

$$
a_{k}\left(\phi_{\text {tilt }}\right)=10^{\frac{G_{\max }}{10}-1.2\left(\frac{\theta_{k}}{\theta_{3 d B}}\right)^{2}-1.2\left(\frac{\phi_{k}-\phi_{t i l t}}{\phi_{3 d B}}\right)^{2}}
$$

$\beta_{k}\left(\phi_{\text {tilt }}\right)$ is a function of antenna boresight angle can be defined as:

$$
\beta_{k}\left(\phi_{t i l t}\right)=\xi_{k} a_{k}\left(\phi_{t i l t}\right) \cdot P L
$$

Where $\xi_{k}$ is the log-normal shadowing fading coefficient [14], $P L$ can be $P L_{L O S}$ or $P L_{N L O S}$ which we defined before.

\section{Simulation Results}

In this section,we illustrate some simulation results to support the correctness of our propositions. A typical 3D scenario in practice we considered in this simulation-in a large building with 12 floors(each floor has $3 \mathrm{~m}$ height). Multiple users randomly distributed in this area. These assumption allows us to investigate the affect from vertical factors and examine the system performance. We fix the $\theta_{3 d B}=70^{\circ}$ and $\phi_{3 d B}=15^{\circ}, A_{m}=20 d B$. To make it simple, a 3D nested distributed Massive MIMO is generated with 8 densely placed antennas and 14 sparsely placed antennas. Based on the definition of 3D nested array, a large system with totally 126 physical and virtual antennas created.

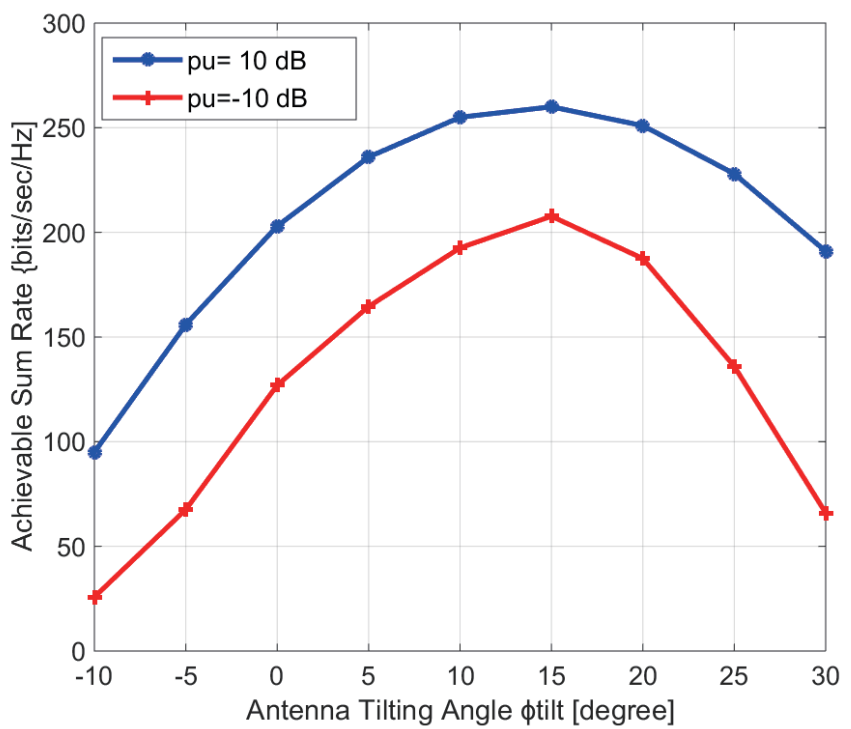

Fig. 5. Achievable sum rate versus antenna tilting angle in different SNR

Since the antenna tilting angle is an important factor which not appeared in 2D case, it is necessary to investigate its affect. Fig. 5 shows the affect of the antenna tilting angle to spectral efficiency. In this simulation, system reach highest spectral efficiency with the tilting angle around $10^{\circ}$. And under lower SNR, the tilting angle has larger negative affect to system performance. So the dynamic adaption on tilting angles may bring some performance improvement in 3D cases.

Fig. 6 shows the achievable sum rate with different number of antennas and placement schemes. Because we use 22

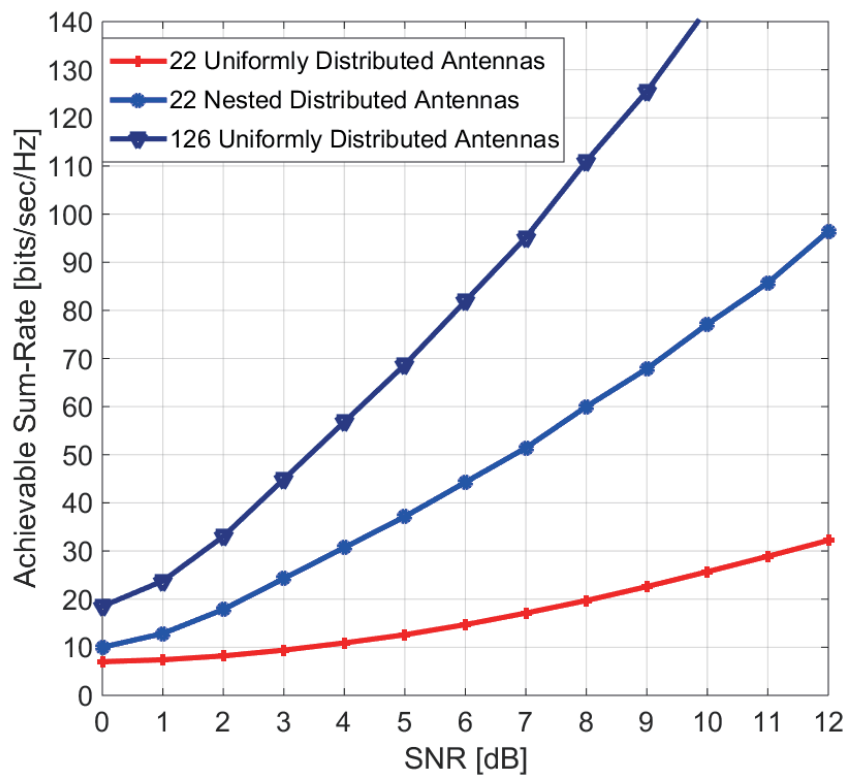

Fig. 6. Different number of antennas and placement schemes versus SNR

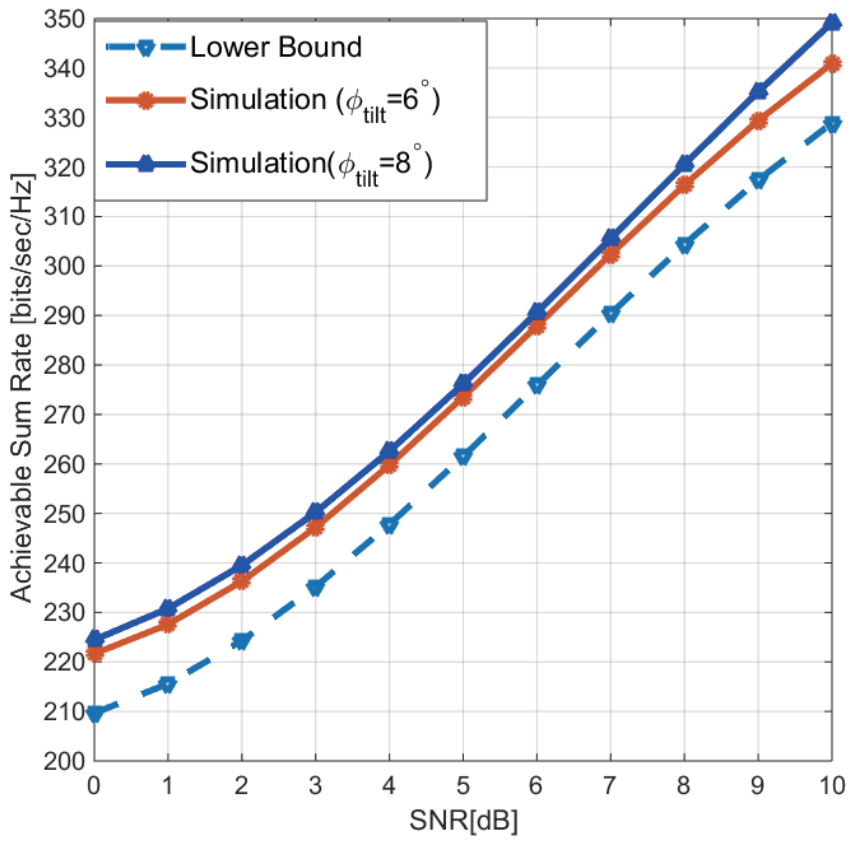

Fig. 7. Achievable sum rate of different tilting angle and lower bound

physical antennas to generate this nested antenna array with totally 126 physical plus virtual antennas, so we also simulate a system with 126 physical uniformly distributed antennas to make a comparison. According to the numerical results, with the same number of physical antennas, our scheme could extremely improve the system performance on sumrate capacity. In other word, to reach the same achievable sum rate, our scheme uses much less antennas. But compared to the system that the number of physical antennas equals to nested antenna array(physical+virtual), the nested Massive 
MIMO system cannot get a such high sum rate, due to the limitation of virtual antennas(interference, complexity, power allocation, .etc).

To capture the effect of antenna boresight angle $\phi_{(t i l t)}$ to achievable sum rate, in Fig. 7, first the lower bound sum rate is compared with simulated sum rate. In this simulation, we set the $M$ equals to 22 as before with the same placement. Two typical value of tilting angle $\phi=6^{\circ}$ and $\phi=8^{\circ}$ is chosen in this experiment, it shows the correctness of another simulation in fig. 5 which is system would reach highest spectral efficiency as tilting angle approaching $10^{\circ}$. As the $\left\|\phi_{(t i l t)}-10^{\circ}\right\|$ increasing, the system sum rate will approach the theoretical lower bound.

\section{CONCLUSION}

This paper firstly extends the dimension from 2D nested array to $3 \mathrm{D}$. Linked it to the cellular communication, a three dimensional nested distributed Massive MIMO system is introduced. We also given an example in section III which use 60 physical generates a nested distributed Massive MIMO system with more than 300 elements. A 3D nested distributed Massive MIMO system model is built. By taking the characteristic of cross difference co-array, we are able to calculate the sumrate capacity of this system. It shows that our scheme could improve the spectral efficiency and also to build a Massive MIMO system with much less antennas. The simulation results are also performed to support the correctness of the statements.

\section{REFERENCES}

[1] P. Pirinen, "A brief overview of $5 \mathrm{~g}$ research activities," in $5 G$ for Ubiquitous Connectivity (5GU), 2014 1st International Conference on, pp. 17-22, IEEE, 2014

[2] H. Shimodaira, G. K. Tran, K. Sakaguchi, and K. Araki, "Investigation on millimeter-wave spectrum for 5g," IEEE, 2015.

[3] J. Qiao, X. Shen, J. Mark, Q. Shen, Y. He, and L. Lei, "Enabling deviceto-device communications in millimeter-wave $5 \mathrm{~g}$ cellular networks," Communications Magazine, IEEE, vol. 53, no. 1, pp. 209-215, 2015.

[4] R. Baldemair, T. Irnich, K. Balachandran, E. Dahlman, G. Mildh, Y. Selen, S. Parkvall, M. Meyer, and A. Osseiran, "Ultra-dense networks in millimeter-wave frequencies," Communications Magazine, IEEE, vol. 53, no. 1, pp. 202-208, 2015.

[5] E. Larsson, O. Edfors, F. Tufvesson, and T. Marzetta, "Massive mimo for next generation wireless systems," Communications Magazine, IEEE, vol. 52, no. 2, pp. 186-195, 2014.

[6] V. Jungnickel, K. Manolakis, W. Zirwas, B. Panzner, V. Braun, M. Lossow, M. Sternad, R. Apelfrojd, and T. Svensson, "The role of small cells, coordinated multipoint, and massive mimo in $5 \mathrm{~g}$," Communications Magazine, IEEE, vol. 52, no. 5, pp. 44-51, 2014.

[7] X. Gao, O. Edfors, F. Rusek, and F. Tufvesson, "Massive mimo performance evaluation based on measured propagation data," Wireless Communications, IEEE Transactions on, vol. 14, no. 7, pp. 3899-3911, 2015.

[8] J. Choi, D. J. Love, and T. Kim, "Trellis-extended codebooks and successive phase adjustment: A path from lte-advanced to fdd massive mimo systems," Wireless Communications, IEEE Transactions on, vol. 14, no. 4, pp. 2007-2016, 2015.

[9] Z. Jiang, A. F. Molisch, G. Caire, and Z. Niu, "Achievable rates of fdd massive mimo systems with spatial channel correlation," Wireless Communications, IEEE Transactions on, vol. 14, no. 5, pp. 2868-2882, 2015.

[10] L. Dai, "An uplink capacity analysis of the distributed antenna system (das): from cellular das to das with virtual cells," Wireless Communications, IEEE Transactions on, vol. 13, no. 5, pp. 2717-2731, 2014.
[11] C.-X. Wang, F. Haider, X. Gao, X.-H. You, Y. Yang, D. Yuan, H. Aggoune, H. Haas, S. Fletcher, and E. Hepsaydir, "Cellular architecture and key technologies for $5 \mathrm{~g}$ wireless communication networks," Communications Magazine, IEEE, vol. 52, no. 2, pp. 122-130, 2014.

[12] Q.-U.-A. Nadeem, A. Kammoun, M. Debbah, and M.-S. Alouini, "3d massive mimo systems: modeling and performance analysis," Wireless Communications, IEEE Transactions on, vol. 14, no. 12, pp. 6926-6939, 2015.

[13] S. Wu, C.-X. Wang, E.-H. M. Aggoune, M. M. Alwakeel, and Y. He, "A non-stationary 3-d wideband twin-cluster model for $5 \mathrm{~g}$ massive mimo channels," Selected Areas in Communications, IEEE Journal on, vol. 32, no. 6, pp. 1207-1218, 2014.

[14] P. Pal and P. Vaidyanathan, "Nested arrays: a novel approach to array processing with enhanced degrees of freedom," Signal Processing, IEEE Transactions on, vol. 58, no. 8, pp. 4167-4181, 2010.

[15] P. Pal and P. Vaidyanathan, "Nested arrays in two dimensions, part i: geometrical considerations," Signal Processing, IEEE Transactions on, vol. 60, no. 9, pp. 4694-4705, 2012.

[16] P. Pal and P. Vaidyanathan, "Nested arrays in two dimensions, part ii: Application in two dimensional array processing," Signal Processing, IEEE Transactions on, vol. 60, no. 9, pp. 4706-4718, 2012.

[17] A. Goldsmith, Wireless communications. Cambridge university press, 2005.

[18] R. Itu, “ $\{$ ITU-R M. 2135: Guidelines for evaluation of radio interface technologies for IMT-Advanced \}," 2008

[19] A. Jassal, H. Khanfir, and S. Martinez Lopez, "Preliminary system-level simulation results for the $3 \mathrm{gpp} 3 \mathrm{~d}$ mimo channel model," in Vehicular Technology Conference (VTC Fall), 2014 IEEE 80th, pp. 1-5, IEEE, 2014.

[20] A. Kuchar, J.-P. Rossi, and E. Bonek, "Directional macro-cell channel characterization from urban measurements," Antennas and Propagation, IEEE Transactions on, vol. 48, no. 2, pp. 137-146, 2000.

[21] J. Fuhl, J.-P. Rossi, and E. Bonek, "High-resolution 3-d direction-ofarrival determination for urban mobile radio," Antennas and Propagation, IEEE Transactions on, vol. 45, no. 4, pp. 672-682, 1997.

[22] H. Q. Ngo, E. G. Larsson, and T. L. Marzetta, "Energy and spectral efficiency of very large multiuser mimo systems," Communications, IEEE Transactions on, vol. 61, no. 4, pp. 1436-1449, 2013.

[23] X. Li, L. Li, and L. Xie, "Achievable sum rate analysis of zf receivers in 3d mimo systems.," TIIS, vol. 8, no. 4, pp. 1368-1389, 2014.

[24] M. Matthaiou, C. Zhong, M. R. McKay, and T. Ratnarajah, "Sum rate analysis of zf receivers in distributed mimo systems," Selected Areas in Communications, IEEE Journal on, vol. 31, no. 2, pp. 180-191, 2013. 